INPLASY

PROTOCOL

To cite: Li et al. A meta analysis of randomized controlled trials on the effectiveness of sandplay therapy. Inplasy protocol 202140095. doi:

10.37766/inplasy2021.4.0095

Received: 18 April 2021

Published: 18 April 2021

Corresponding author: Jinde Li

lijinde198526@163.com

Author Affiliation:

School of Education Science, Guangxi University for

Nationalities

Support: Guangxi (2021JD004/2020LSZ069).

Review Stage at time of this submission: Preliminary searches.

Conflicts of interest:

None declared.

\section{A meta analysis of randomized controlled trials on the effectiveness of sandplay therapy}

Li, J1; Wang, J2; Jiang, Y3; Ji, S4.

Review question / Objective: What is the effectiveness of sandplay therapy? Who can benefit most from sandplay therapy? For which outcomes is sandplay therapy most effective?

Condition being studied: Sandplay therapy is an expressive arts therapy for healing and transformative work that is rooted in the theories of Dora Kalff and C.G. Jung, which has proven effective for a broad variety of emotional difficulties. However, there are still few meta-analysis of randomized controlled trials for sandplay thepray.

Information sources: PsycINFO, PsycARTICLES, CINAHL (EbscoHost) , PubMed, Scopus, Web of Science , Embase, MEDLINE(R), CENTRAL, CNKI, WANFANGdata, CQVIP.

INPLASY registration number: This protocol was registered with the International Platform of Registered Systematic Review and Meta-Analysis Protocols (INPLASY) on 18 April 2021 and was last updated on 18 April 2021 (registration number INPLASY202140095).

\section{INTRODUCTION}

Review question / Objective: What is the effectiveness of sandplay therapy? Who can benefit most from sandplay therapy? For which outcomes is sandplay therapy most effective?
Condition being studied: Sandplay therapy is an expressive arts therapy for healing and transformative work that is rooted in the theories of Dora Kalff and C.G. Jung, which has proven effective for a broad variety of emotional difficulties.However, there are still few meta-analysis of 
randomized controlled trials for sandplay thepray.

\section{METHODS}

Participant or population: There are no strict restrictions.

Intervention: Sandplay therapy.

Comparator: Studies can include a nonexposed control group and/or an experimental group using another psychological intervention.

Study designs to be included: Randomized controlled trials.

Eligibility criteria: Only papers published in peer-reviewed journals would be included, if reporting findings from randomised controlled trials studies. Conference papers; or unpublished data; as well as papers reporting findings of nonexperimental, observational studies will be excluded.

Information sources: PsycINFO, PsycARTICLES, CINAHL (EbscoHost), PubMed, Scopus, Web of Science, Embase, MEDLINE (R) , CENTRAL, CNKI, WANFANGdata, CQVIP.

Main outcome(s): Any extractable psychological and behavioral outcomes.

Data management: All retrieved titles are first deduplicated. Two reviewers independently assess the remaining titles and abstracts against the inclusion and exclusion criteria. After that, the reviewers assess the full texts of the remaining literature. A standardized form will be used for data extraction which will be conducted independently by two reviewers. Any disagreement in data selection and extraction is resolved by discussion.

Quality assessment / Risk of bias analysis: Risk of bias of the included studies will be assessed with the Cochrane Collaboration Guidelines in randomization, allocation concealment, blinding of therapists, blinding of participants, blinding of outcome assessors, handling of incomplete data, selective reporting and any other risk of bias.

Strategy of data synthesis: Aggregate trial data will be collected and a Meta-Analysis will be carried out using STATA 14 or Comprehensive Meta Analysis 3. Separate meta-analyses will be conducted for different outcomes from included studies. Fixed effects models will be used whenever the heterogeneity is low to medium $\left(I^{2} 50\right)$, random effects models will be used instead. The standardized mean difference (SMD) will be computed as the effect size. Publication bias will be examined by visual inspection of funnel plots and the Egger's regression test.

Subgroup analysis: Participants will be grouped by Region, age, and interventions format (group vs individual), if there are sufficient studies.

Sensitivity analysis: Sensitivity analyses will be conducted to test the robustness of studies according to the quality of method and the sample size.

Language: Without any language restrictions.

Country(ies) involved: China.

Keywords: sandplay therapy; effectiveness; meta analysis; RCT.

Contributions of each author:

Author 1 - Jinde Li.

Author 2 - Jue Wang.

Author 3 - Yuxing Jiang.

Author 4 - Shuning Ji. 